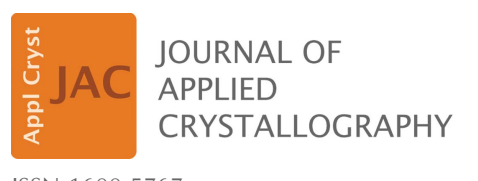

ISSN 1600-5767

Received 11 February 2020

Accepted 25 March 2020

Edited by S. Boutet, SLAC National Accelerator Laboratory, Menlo Park, USA

Keywords: variable temperature; X-ray scattering; powder X-ray diffraction; sample environments; negative thermal expansion.

Supporting information: this article has supporting information at journals.iucr.org/j

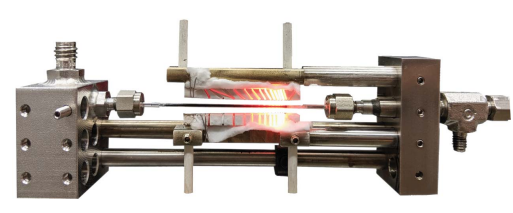

C 2020 International Union of Crystallography

\section{A thermal-gradient approach to variable- temperature measurements resolved in space}

\author{
Daniel $\mathrm{O}^{\prime}$ Nolan, ${ }^{\mathrm{a}}$ Guanglong Huang, ${ }^{\mathrm{b}}$ Gabrielle E. Kamm, ${ }^{\mathrm{a}}$ Antonin Grenier, ${ }^{\mathrm{a}}$ \\ Chia-Hao Liu, ${ }^{\text {c }}$ Paul K. Todd, ${ }^{d}$ Allison Wustrow, ${ }^{\text {d }}$ Gia Thinh Tran, ${ }^{d}$ David Montiel, ${ }^{\text {b }}$ \\ James R Neilson, ${ }^{\mathrm{d}}$ Simon J. L. Billinge, ${ }^{\mathrm{c}}$ Peter J. Chupas, ${ }^{\text {a }}$ Katsuyo S. Thornton ${ }^{\mathrm{b}}$ and \\ Karena W. Chapman ${ }^{\mathrm{a} *}$
}

\footnotetext{
a Department of Chemistry, Stony Brook University, 100 Nicolls Road, Stony Brook, New York 11794, USA, ${ }^{\mathbf{b}}$ Department of Materials Science and Engineering, University of Michigan, Ann Arbor, Michigan 48109, USA, 'Department of Materials Science and Engineering, Columbia University, New York, New York 10027, USA, and d Department of Chemistry, Colorado State University, Fort Collins, Colorado 80523, USA. *Correspondence e-mail:

karena.chapman@stonybrook.edu
}

Temperature is a ubiquitous environmental variable used to explore materials structure, properties and reactivity. This article reports a new paradigm for variable-temperature measurements that varies the temperature continuously across a sample such that temperature is measured as a function of sample position and not time. The gradient approach offers advantages over conventional variable-temperature studies, in which temperature is scanned during a series measurement, in that it improves the efficiency with which a series of temperatures can be probed and it allows the sample evolution at multiple temperatures to be measured in parallel to resolve kinetic and thermodynamic effects. Applied to treat samples at a continuum of temperatures prior to measurements at ambient temperature, the gradient approach enables parametric studies of recovered systems, eliminating temperaturedependent structural and chemical variations to simplify interpretation of the data. The implementation of spatially resolved variable-temperature measurements presented here is based on a gradient-heater design that uses a 3Dprinted ceramic template to guide the variable pitch of the wire in a resistively heated wire-wound heater element. The configuration of the gradient heater was refined on the basis of thermal modelling. Applications of the gradient heater to quantify thermal-expansion behaviour, to map metastable polymorphs recovered to ambient temperature, and to monitor the time- and temperaturedependent phase evolution in a complex solid-state reaction are demonstrated.

\section{Introduction}

Temperature is arguably the most universally explored environmental variable for non-ambient studies of materials structure. X-ray scattering and spectroscopic measurements as a function of temperature are applied to probe temperaturedependent structure parameters, transitions, structureproperty relationships, and reaction mechanisms and kinetics (Reiss, 2018; Evans \& Evans, 2004). Traditionally, temperature-dependent studies have collected data as a sample is progressively heated or cooled, either continuously or in discrete steps. In practice, these measurements are limited by how fast the temperature can be changed, the temperature range and the number of temperature points of interest. Timedependent measurements at several fixed temperatures are often used to explore reaction kinetics and to quantify kinetic parameters and activation energies (Millange et al., 2000).

Here we present a new paradigm for high-throughput variable-temperature experiments, wherein a continuous 
temperature gradient is generated across a uniform sample. Data are collected as a function of position, over which temperature varies, rather than as a function of time/ temperature. We developed a controlled-temperaturegradient sample environment based on our versatile flow-cell/ furnace design (Chupas et al., 2008), wherein the resistive heating elements were adapted to produce a thermal gradient rather than spatially uniform heating. The temperature resolution depends on the spatial resolution of the X-ray measurements and the slope of the thermal gradient. The thermal-gradient heater provides advantages in the efficiency with which variable-temperature data can be collected, eliminating the ramping and equilibration time.

We demonstrate the applicability of this approach for studying temperature-dependent structural parameters, phase transitions and solid-state reactions. We show that it can be used in situ to accelerate variable-temperature studies or to monitor the time evolution of a system at a continuum of temperatures in parallel. Alternatively, it can enable parametric studies to explore the effect of heating at different temperatures by mapping the capillary-loaded sample following recovery to ambient temperature.

\section{Gradient-heater design and performance}

\subsection{Gradient-heating element}

A widely used flow-cell/furnace reported previously (Chupas et al., 2008) was used as the basis for the new gradient-heater design. The flow-cell/furnace has a modular architecture: compression fittings that hold the sample-loaded capillary and heating elements are assembled with precisionground rods and precisely matched mounting blocks. This design uses resistively heated wire-wound elements mounted above and below the sample capillary to heat the sample on the basis of feedback from a thermocouple inside the capillary. The heating elements are fabricated by winding Kanthal (FeCrAl alloy) wire around a ceramic bar. Stable uniform heating of the sample is facilitated by a uniform winding of the heating wire, radiative heating by the ceramic rod inside the wire coil, and the use of heat shields that minimize air currents and convective heat loss.

The gradient-heater design adapts the heating elements of the original flow-cell/furnace, to non-uniformly heat the sample, by progressively varying the pitch of the resistive wire in the heating element (Fig. 1). The heating element is extended by $50 \%$ relative to the original heater to facilitate measurements over a broader temperature range or with finer temperature resolution. While the wire pitch could be tailored to deliver an arbitrary temperature profile, for the purpose of this initial report, we target the simplest case of an approximately linear monotonic temperature gradient. At one edge of the element, a short region of uniform high-density winding delivers the maximum temperature. The pitch of the resistivewire windings is then increased continuously, providing an approximately linear decrease in temperature [Fig. 1(a)]. The gradient heaters tested here varied the winding pitch between 12 and 1 turns per centimetre.

The variations in winding pitch of the resistive wire are controlled by grooves in the central ceramic bar. Here, the silica-based ceramic bar was fabricated using 3D printing (Ceramic Resin, Formlabs), which affords a high degree of control and reproducibility. The ceramic is electrically insulating with high thermal and mechanical stability (over $1000^{\circ} \mathrm{C}$ reported, up to at least $1300^{\circ} \mathrm{C}$ in our tests). While the original elements used commercially available cylindrical rods, the gradient design uses a rectangular bar (cross section $4 \times$ $8 \mathrm{~mm}$ ). This rectangular geometry helps maintain the tension

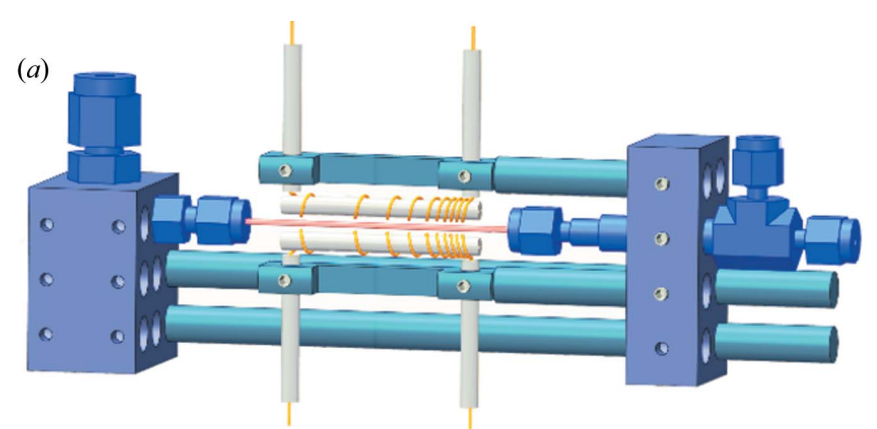

(b)

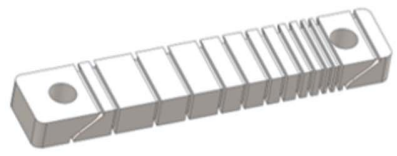

(c)

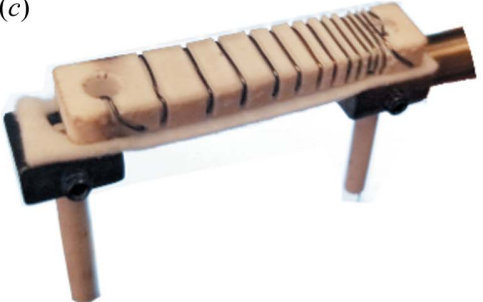

(d)

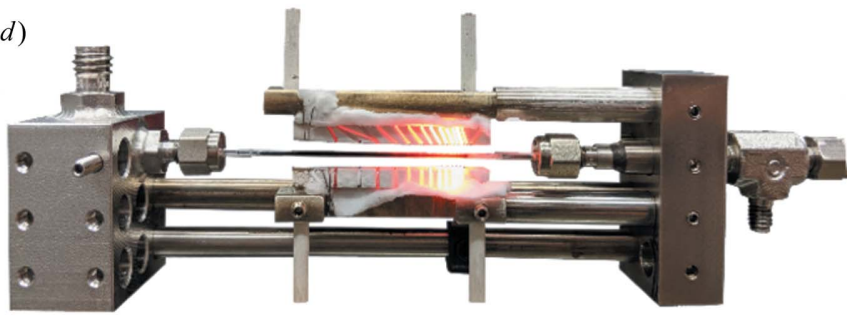

(e)

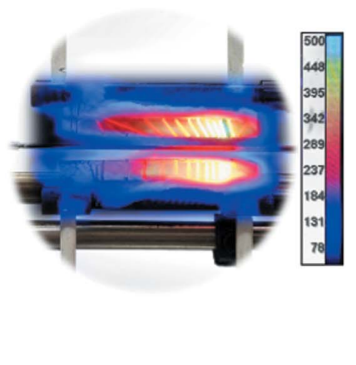

Figure 1

CAD and photographic representations of the flow-cell/furnace-equipped gradient heater $(a)$ and $(d),(b)$ the ceramic bar that guides the spacing of the resistive wire, and $(c)$ a single gradient heater. Black-body radiation from the resistive wires serves as an intuitive indicator of the thermal gradient. $(e) \mathrm{A}$ thermal image of the gradient heater overlaid on a photograph of the heating zone (colour bar shows the temperature in ${ }^{\circ} \mathrm{C}$ ). 
when fabricating the element, provides a wider heating zone perpendicular to the thermal gradient and further reduces air currents, which have been identified as a principal source of thermal fluctuation in related heaters (Newton et al., 2019).

The gradient heaters are fixed to stainless-steel holders [Fig. 1(c)]; a pair of ceramic tubes (Omega Engineering Inc., part No. DH-1-24-100) are mounted in the stainless-steel holders to sheathe the resistive wire and ensure reproducible positioning of the ceramic bar and gradient. Alumina felt (Zircar Ceramics) placed between the resistive-wire coil and stainless-steel holders prevents shorting. The ceramic tubes can support vertically mounted wires which serve as fiducial markers for positioning the thermal gradient within the X-ray beam. A hole in the heater ceramic fixes the thermocouple position relative to the thermal gradient. These measures to ensure reproducible positioning of the sample, heating elements and thermocouple tip are critical to calibrating the temperature at all positions across the gradient.

\subsection{Assembly}

Sample-loaded capillaries of $c a 1.1 \mathrm{~mm}$ outer diameter were assembled in the flow-cell/furnace. The length of sample in the capillary was increased from $\mathrm{ca} 5 \mathrm{~mm}$ in a typical conventional setup to $c a 35-40 \mathrm{~mm}$ in the gradient heater (Fig. 1). Resistive heating of the gradient elements, fitted with 28 -gauge wire and mounted above and below the sample, was achieved with a DC power source (BK Precision XLN3640) in combination with a temperature controller (Stanford Research Systems PTC10) on the basis of feedback from a K-type thermocouple at a fixed position within the gradient. Heat shields minimized air currents and associated thermal fluctuations.

\subsection{Temperature calibration}

The sample temperature at different positions along the thermal gradient was determined either using a K-type thermocouple inside an empty capillary or on the basis of lattice parameters determined from X-ray diffraction data collected
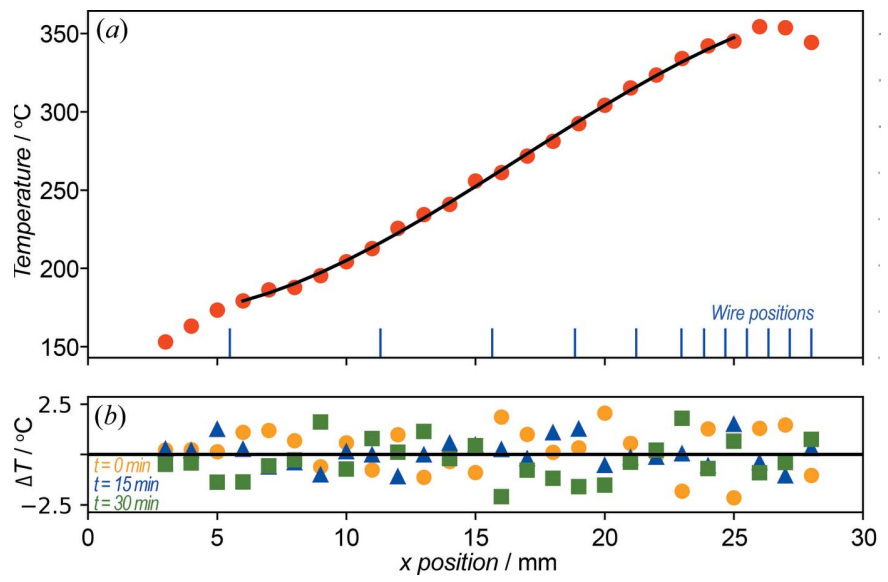

Figure 2

(a) An example of a thermal gradient generated by variable spacing of resistive wire (indicated as blue lines). (b) The deviation in the temperature from a polynomial fit (black line) for a series of measurements separated by $15 \mathrm{~min}$ (circles, triangles, squares). for a mixture of solids with different, known coefficients of thermal expansion (Drews, 2000).

As an example, a capillary containing a mixture of $\mathrm{NaCl}$ and $\mathrm{Si}$ was assembled within the gradient heater. X-ray scattering data were collected at beamline 11-ID-B of the Advanced Photon Source with an X-ray wavelength of $0.2115 \AA$ and beamline 28-ID-1 of the National Synchrotron Light Source II (NSLS-II) with an X-ray wavelength of $0.1668 \AA$ A. Data were collected using a $0.5 \times 0.5 \mathrm{~mm}$ beam at $1 \mathrm{~mm}$ intervals along the thermal gradient. The temperature-dependent lattice parameters for each phase were quantified via a Le Bail fit to the data within TOPAS (Coelho, 2018). The temperature at each point within the gradient was estimated from the observed offset between the lattice parameters for the phases and their known room-temperature lattice parameters and coefficients of thermal expansion (Drews, 2000; Stinton \& Evans, 2007).

A linear variation in the pitch of the resistive wire yielded an approximately linear thermal gradient across the sample (Fig. 2), the wire-winding density correlating with observed temperature. The stability of the temperature across the thermal gradient was evaluated from repeated temperaturecalibration data collected at $15 \mathrm{~min}$ intervals. The temperature was stable and did not deviate from a fit to all data by more than $\pm 2^{\circ}$ (Fig. 2). There was no time-dependent trend observed in the temperature deviations. We hypothesize that heating of the ceramic bar by the wire and the interplay with the thermal conductivity of the ceramic play an important role in smoothing the temperature - the heated ceramic serves as a heat reservoir that also contributes to sample heating. The mass of the sample is small relative to the heater and ceramic, so exo- and endothermic processes within the sample are unlikely to lead to significant or sustained local deviations in the temperature.

A linear relationship between the maximum temperature $\left(T_{\max }\right)$ and the temperature range $\left(T_{\text {range }}\right)$ and minimum $\left(T_{\min }\right)$ temperature (Fig. 3) was observed.

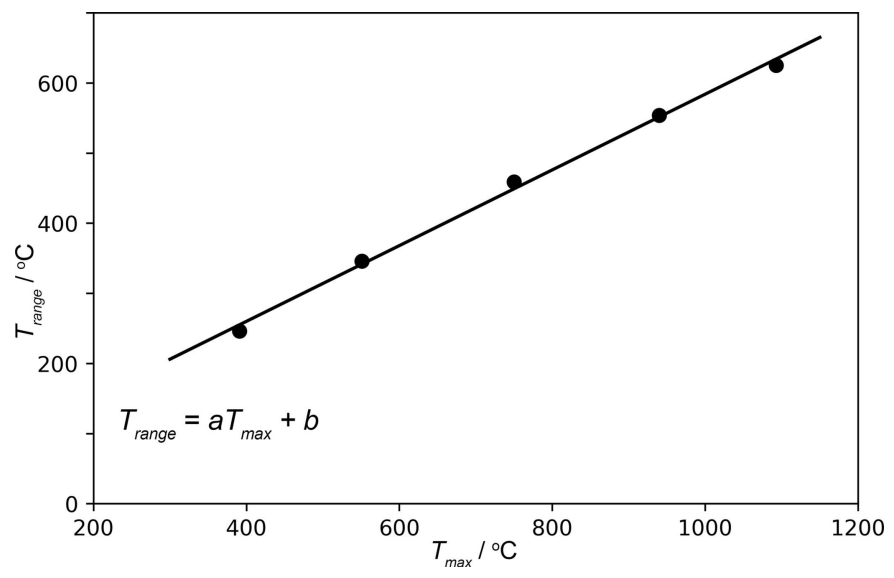

Figure 3

For a given gradient-heater configuration the temperature range spanned by the gradient heater increases linearly with the maximum temperature. The relationships are given as $T_{\min }=(1-a) T_{\max }-b$ or $T_{\text {range }}=$ $a T_{\max }+b$. For the present gradient-heater configuration (shown in Fig. 2) $a=0.54, b=44^{\circ} \mathrm{C}$. 


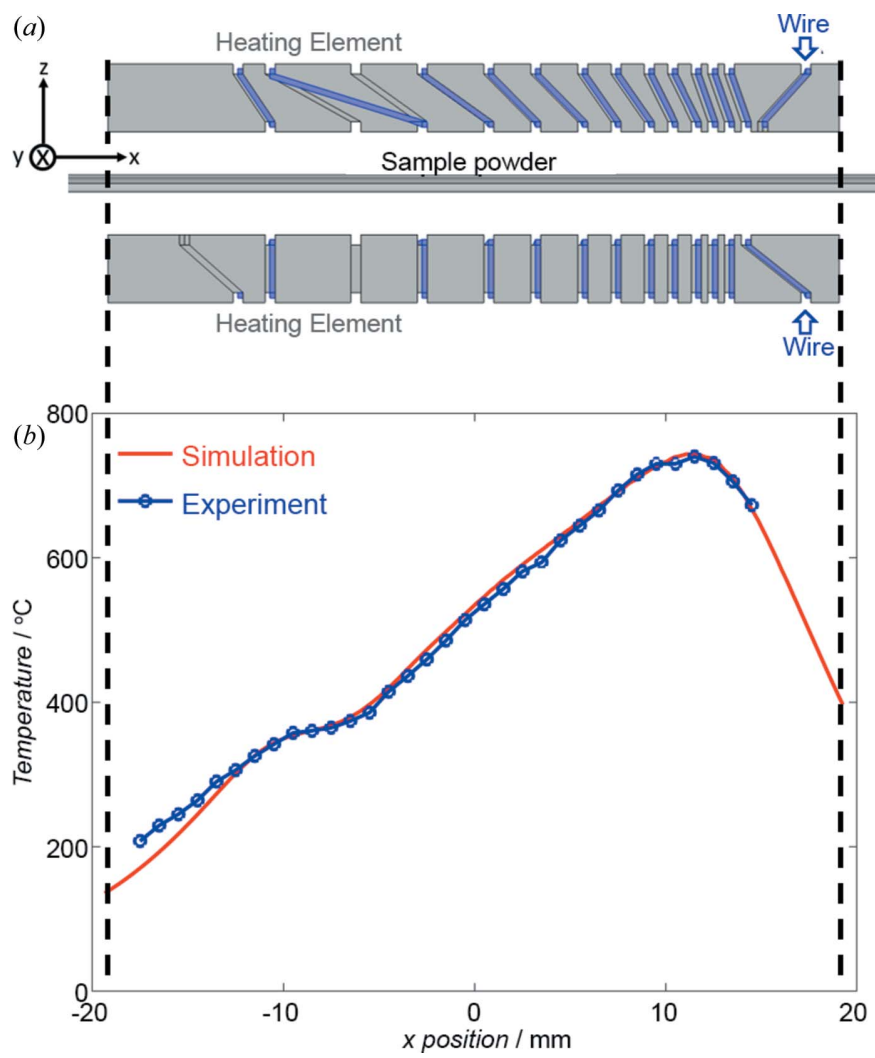

Figure 4

(a) The model geometry. As drawn, the top and bottom heating elements show views of the two different element edges (i.e. they are related by a $180^{\circ}$ rotation about the $x$ axis). Grooves that face the sample are aligned parallel to the $\mathbf{y}$ direction. (b) The simulated (red) and experimental (blue) temperature profiles. The edges of the heating elements are indicated by the vertical dashed lines.

\section{Thermal modelling}

\subsection{Model parametrization}

To supplement thermal measurements and aid the heatingelement design, a parameterized thermal transfer model was applied to simulate the steady-state temperature profile within a powder sample heated by the heating elements, each of which consists of the resistive wire and central ceramic bar, using COMSOL Multiphysics version 5.4 (COMSOL AB,
Stockholm, Sweden; https://www.comsol.com). Parameters used herein (see supporting information, Table S1) were obtained from the literature and experimental data described in the supporting information. The simulation geometry consisted of an $\mathrm{NaCl} / \mathrm{Si}$ sample powder mixture placed between two ceramic bars with heating wires wound around them [Fig 4(a)]. As current is applied through the wires, they undergo Joule heating, which is transferred to the sample and ceramic. The simulated sample temperature values are averaged along the direction of the X-ray beam, which is along the $\mathbf{y}$ direction in Fig. 4(a). The resulting temperature profile is shown in Fig. 4(b), which matches the experimental data.

\subsection{Controlling the temperature profile}

The temperature range spanned by the gradient heater depends on the degree to which the wire spacing varies across the heater. A larger variation in the wire spacing corresponds to a greater temperature range for the same maximum temperature. Three simulations were conducted to examine the temperature profile when the resistive wires are configured differently [Fig. 5(a)]. The heating element for Mark 2A has a smaller variation in the wire spacing (with a maximum distance, $D$, of $6.7 \mathrm{~mm}$ between the centres of two grooves) than that for Mark 2B $(D=8.2 \mathrm{~mm})$, followed by Mark 2C $(D=11.4 \mathrm{~mm})$. The minimum distances between the centres of two grooves for the three heating elements are identical. Detailed schematics of the three winding configurations are shown in Fig. S1. The simulation suggests that a greater temperature span can be achieved with a larger variation in the wire spacing.

Simulations were then undertaken to explore the impact of the ceramic bar width on the temperature profile. The simulated temperature profiles [Fig. 5(b)] suggest that a wider surface of ceramic bar affords a smoother, more uniform, temperature profile along the sample. This effect is probably due to a smoother temperature profile along the centre line of the surface of the ceramic bar facing the sample, afforded by the greater distance from the sides of the ceramic bar where heat loss occurs. We examined the temperature profile of the sample when the sample is offset from the centre of the gradient heater [Fig. 5(c)]. Offsets of up to $1 \mathrm{~mm}$ result in

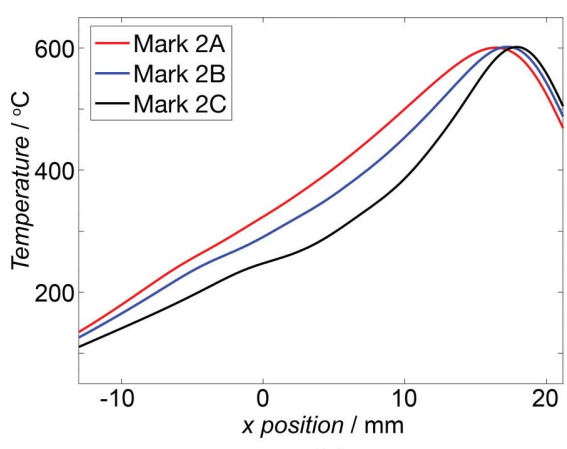

(a)

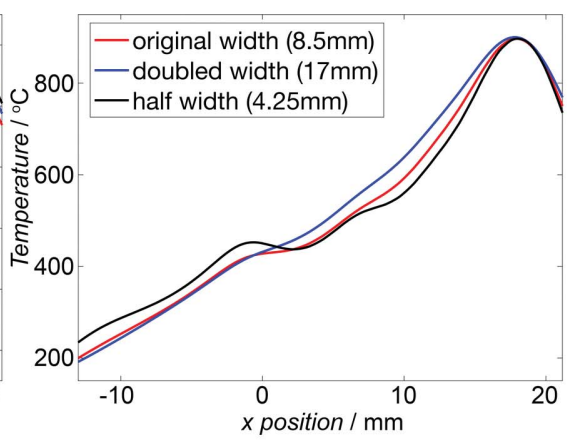

(b)

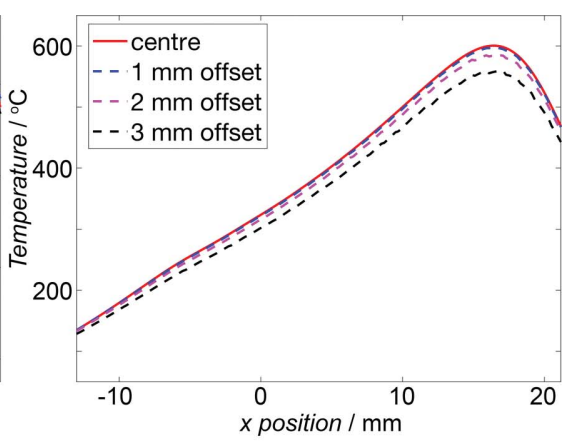

(c)

Figure 5

The simulated temperature profile of the sample between two ends of the resistive wire comparing $(a)$ different winding configurations, $(b)$ different widths of the heating elements (Mark 2C), and (c) the effect of the sample being offset in the $\mathbf{y}$ direction (Mark 2A). 
negligible change in the temperature profile. This offset is much larger than the error in alignment permitted by the cell design, demonstrating that a small misalignment would not alter the temperature profile of the sample. By contrast, temperature errors of tens of degrees are observed when the capillary is offset by $3 \mathrm{~mm}$ (i.e. within $1 \mathrm{~mm}$ of the edge of the ceramic bar). This reflects the benefits of the wide ceramic bar, and rectangular profile, for temperature fidelity.

\section{Example applications}

\subsection{Negative thermal-expansion behaviour in $\mathrm{Zn}(\mathrm{CN})_{2}$}

Most materials undergo positive thermal expansion, that is, the material volume increases with temperature. Certain framework materials exhibit the opposite behaviour in that their volume decreases with increasing temperature - a phenomenon referred to as negative thermal expansion (NTE). Such NTE materials are of fundamental and applied interest, with notable applications in compensating for the positive thermal expansion of other materials. Studies of NTE materials as a function of temperature are used to quantify the magnitude of the thermal expansion, expressed as the coefficient of thermal expansion (CTE, $\alpha=\mathrm{d} l / \mathrm{d} T$ ), by monitoring the temperature dependence of the lattice parameters, and to investigate the structural mechanism underlying the behaviour.

Here we use the gradient heater in situ to characterize the NTE behaviour in zinc cyanide, $\mathrm{Zn}(\mathrm{CN})_{2}(P n \overline{3} m, a=$ $\sim 5.865 \AA$ ), in the range $c a 200-700^{\circ} \mathrm{C}$, by quantifying the temperature-dependent lattice parameters and, accordingly, to evaluate the CTE. $\mathrm{Zn}(\mathrm{CN})_{2}$ is a cyanide-bridged framework material that exhibits strong NTE, which is most pronounced at $-93^{\circ} \mathrm{C}$, where $\alpha=-19.8 \times 10^{-6} \mathrm{~K}^{-1}$ (Chapman et al., 2005). $\mathrm{X}$-ray scattering data were collected at beamline 11-ID-B at the Advanced Photon Source using high-energy X-rays $(\lambda=$ $0.2113 \AA$ A). An X-ray beam of $0.5 \times 0.5 \mathrm{~mm}$ was used to probe the sample at $1 \mathrm{~mm}$ intervals along the thermal gradient. This corresponded to temperature intervals of $c a 19^{\circ} \mathrm{C}$. Data were

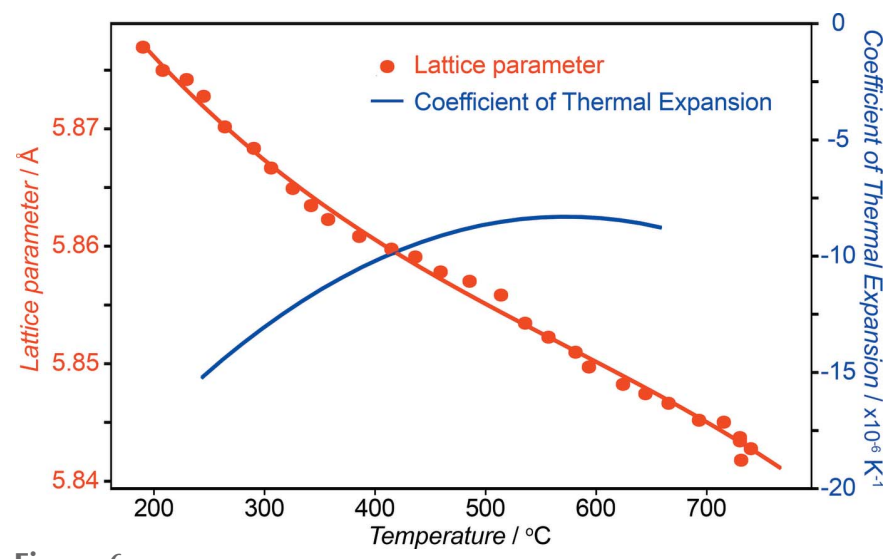

Figure 6

The temperature dependence of the lattice parameters in $\mathrm{Zn}(\mathrm{CN})_{2}$, measured using the gradient heater, and the corresponding coefficients of thermal expansion. collected in $10 \mathrm{~s}$ exposures, at $\mathrm{ca} 40$ positions. The CTE for $\mathrm{Zn}(\mathrm{CN})_{2}$ changes from $\mathrm{ca}-16 \times 10^{-6} \mathrm{~K}^{-1}$ at $208^{\circ} \mathrm{C}$ to plateau at $c a-8 \times 10^{-6} \mathrm{~K}^{-1}$ above $c a 560^{\circ} \mathrm{C}$ (Fig. 6). The magnitude of the CTE at $200^{\circ} \mathrm{C}$ and the decrease in the magnitude of CTE with increasing temperature is consistent with previous reports.

The efficiency with which high-quality variable-temperature powder diffraction data can be collected is notable; in the present example, data spanning a broad temperature range were collected in $<3 \mathrm{~min}$. By comparison, conventional variable-temperature powder X-ray diffraction collection strategies would take $>100$ min to span a similar temperature range (assuming a ramp rate of $5^{\circ} \mathrm{C} \mathrm{min}^{-1}$ ).

\subsection{Structural transitions and polymorphism in $\mathrm{Nb}_{2} \mathrm{O}_{5}$}

Transitions between different polymorphs of a material can be induced through heating, with the potential to recover high-temperature polymorphs as metastable phases at ambient conditions. Variable-temperature X-ray scattering studies are valuable in identifying the stability and metastability of each polymorph, along with the temperature at which transitions occur.

Several $\mathrm{Nb}_{2} \mathrm{O}_{5}$ polymorphs (TT-, T-, B- and $\mathrm{H}_{-} \mathrm{Nb}_{2} \mathrm{O}_{5}$ ) form at temperatures up to $1100^{\circ} \mathrm{C}$ and can be recovered to room temperature as metastable phases (Griffith et al., 2016). The temperature-dependent polymorphism of $\mathrm{Nb}_{2} \mathrm{O}_{5}$, produced through oxidation of $\mathrm{NbO}_{2}$, was documented by mapping the recovered sample capillary following heating in the gradient heater (Fig. 7). The $\mathrm{NbO}_{2}$ precursor was treated in the gradient heater in air for $15 \mathrm{~min}$ at temperatures in the range $200-700^{\circ} \mathrm{C}$. The capillary was recovered to room temperature and X-ray scattering data were collected at beamline 11-ID-B at the Advanced Photon Source using high-energy $\mathrm{X}$-rays $(\lambda=$ $0.2113 \AA$ ). Data were collected at 35 positions at $1 \mathrm{~mm}$ intervals along the capillary using a $0.5 \times 0.5 \mathrm{~mm}$ beam with $0.1 \mathrm{~s}$ exposures.

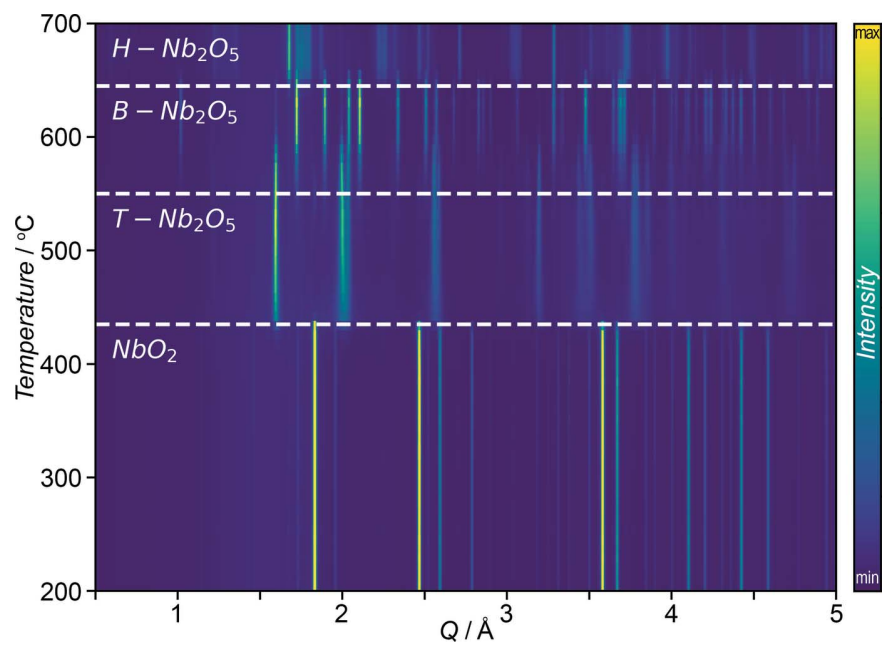

Figure 7

Diffraction data corresponding to the oxidation of $\mathrm{NbO}_{2}$ to different $\mathrm{Nb}_{2} \mathrm{O}_{5}$ polymorphs. Position-dependent data were obtained following recovery of the capillary treated at $200-700^{\circ} \mathrm{C}$. 
Oxidation from black $\mathrm{NbO}_{2}\left(I 4_{1} / a\right)$ to cream-coloured $\mathrm{T}^{-\mathrm{Nb}_{2}} \mathrm{O}_{5}$ (Pbam) is observed at $\mathrm{ca} 425^{\circ} \mathrm{C}$, followed by the formation of grey $\mathrm{B}-\mathrm{Nb}_{2} \mathrm{O}_{5}(\mathrm{C} 2 / \mathrm{c})$ at $\mathrm{ca} 550^{\circ} \mathrm{C}$, and the observation of disordered white $\mathrm{H}-\mathrm{Nb}_{2} \mathrm{O}_{5}(P 2 / m)$ starting at ca $650^{\circ} \mathrm{C}$. For comparison, Griffith et al. observed the formation of TT- $-\mathrm{Nb}_{2} \mathrm{O}_{5}$ at $300^{\circ} \mathrm{C}$, followed by the T-phase at $600^{\circ} \mathrm{C}$, the B-phase at $850^{\circ} \mathrm{C}$ and the $\mathrm{H}$-phase at $1100^{\circ} \mathrm{C}$, for treatment of individual samples for $24 \mathrm{~h}$. Distinct TT and T phases are not resolved here. The series of transitions found in the present study follow the same sequence as reported by Griffith et al. but are observed at lower temperatures. For example, the $\mathrm{B}$ phase is observed here at $550-650^{\circ} \mathrm{C}$ but is only found at much higher temperature $\left(850^{\circ} \mathrm{C}\right)$ by Griffith et al. We attribute these differences to the shorter time at temperature in the gradient heater $(15 \mathrm{~min} c f .24 \mathrm{~h})$. The shorter heating time will favour the kinetic over the thermodynamic products. In this case, the $\mathrm{B}$ phase is likely to be the kinetic product at 550 $650^{\circ} \mathrm{C}$ but the thermodynamic product at $850^{\circ} \mathrm{C}$. While differences in particle size can also impact phase transition temperatures, these effects are most pronounced for nanoscale materials, and can be excluded here as both the present sample and that reported by Griffith et al. have well defined crystallites consistent with bulk crystallites.

That the measurements on the recovered material are undertaken at the same temperature means that differences in a given phase can be interpreted as differences in the irreversible changes in the structure or chemistry induced at different temperatures, rather than reversible temperaturedependent effects such as thermal expansion or displacive phase transitions. While both increasing crystallite size and reducing disorder can contribute to a sharpening of the diffraction peaks, given the known existence of a lowtemperature disordered phase, 'TT-Nb2O5', which transitions to the ordered $\mathrm{T}$ phase, we propose that the observed peak sharpening reflects a progressive ordering process.

\subsection{Solid-state reaction: metathesis}

Solid-state reactions involve coupled transformations of chemistry and structure that can be complex to disentangle. Insight into the mechanism and pathway for such reactions can be obtained by characterizing how the structure, chemistry and abundance of the multiple crystalline reagents, intermediates, products, impurities and polymorphs thereof evolve as a function of time and reaction temperature using in situ X-ray scattering (Jiang et al., 2017; Martinolich \& Neilson, 2017; Shoemaker et al., 2012; Todd et al., 2019).

For the ternary metathesis reaction $\mathrm{LiMnO}_{2}+\mathrm{YOCl} \rightarrow$ $\mathrm{LiCl}+\mathrm{YMnO}_{3}$, the time evolution of nine discrete crystalline phases was observed at a continuum of temperatures up to $750^{\circ} \mathrm{C}$. Variable-temperature in situ X-ray scattering data were collected at beamline 28-ID-2, XPD $(\lambda=0.1949 \AA)$, at NSLSII, in the gradient heater following isothermal heating for 20 and $40 \mathrm{~min}$, and following cooling to room temperature. Preparation of the reaction precursors has been described previously (Todd et al., 2019).
The scattering data show complex coupled changes in the diffraction peaks as a function of time and temperature (Fig. 8). Non-negative matrix factorization (NMF), implemented using the Python scikit-learn package (Pedregosa et al., 2011), was applied to resolve correlated features in the data as separate components and to quantify their associated weightings (Fig. 8). The use of such blind-signal separation has been established for images (Lee \& Seung, 1999), audio signals (Samaragdis \& Brown, 2003) and diffraction patterns (Long et al., 2009; Kusne et al., 2015; Geddes et al., 2019; Chapman et al., 2015). Each component groups peaks that are

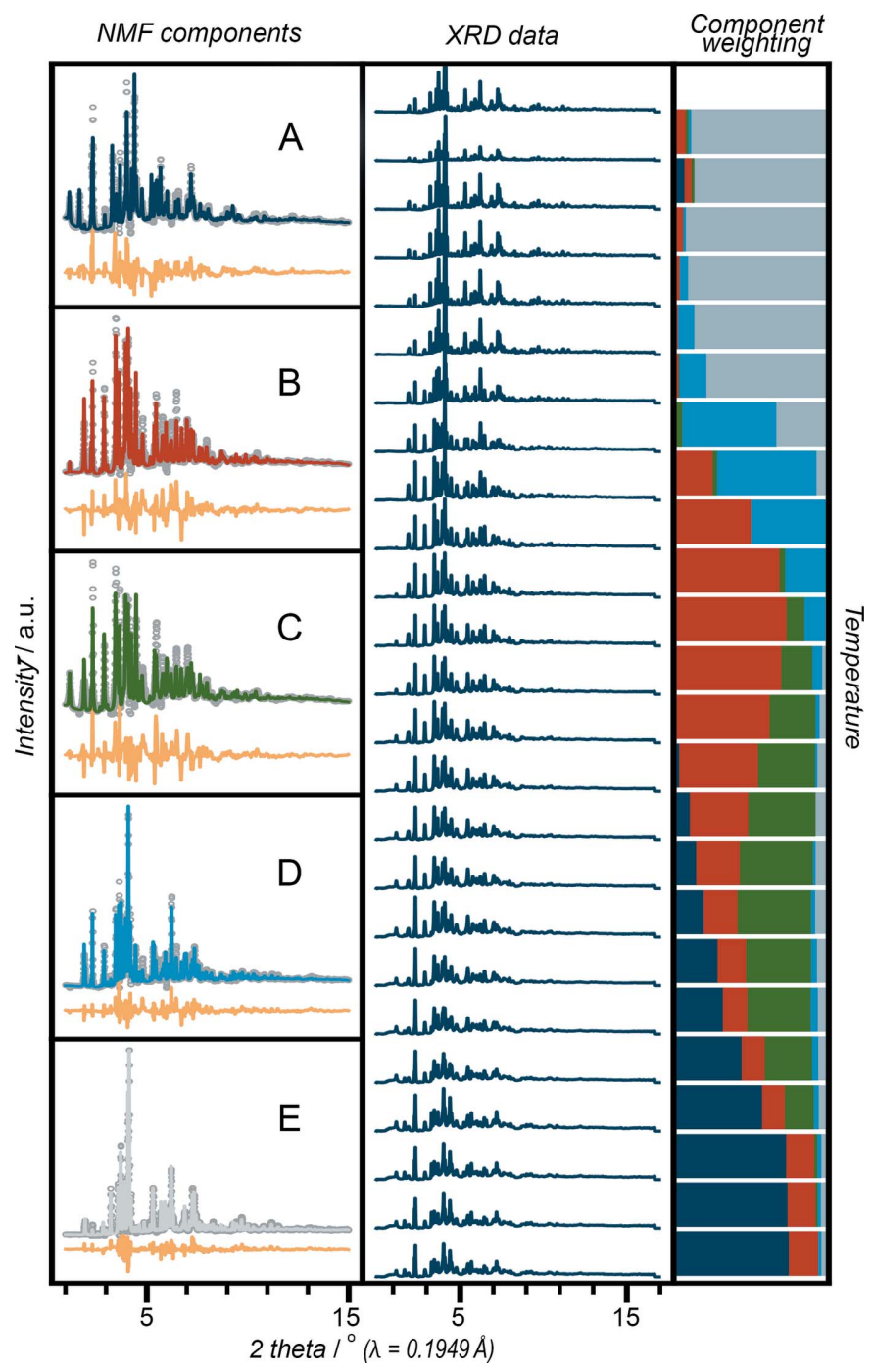

Figure 8

Variable-temperature synchrotron X-ray diffraction data documenting the ternary metathesis reaction collected after $c a 20 \mathrm{~min}$ at a continuum of temperatures: $\mathrm{LiMnO}_{2}+\mathrm{YOCl} \rightarrow \mathrm{YMnO}_{3}+\mathrm{LiCl}$ (middle). The series of variable-temperature data can be described by five components (left), derived from an NMF analysis, with the weightings of the five components changing as a function of temperature, reflecting different states of the metathesis reaction (right). Conventional phase identification and Rietveld refinement applied to the NMF components (left) can identify the multiple phases that contribute to each NMF component and their relative abundance. For example, component A contains $23 \% \mathrm{t}-\mathrm{YOCl}$, $22 \% \mathrm{LiMnO}_{2}, 7 \% \mathrm{r}-\mathrm{YOCl}, 13 \% \mathrm{Y}_{3} \mathrm{O}_{4} \mathrm{Cl}, 6 \% \mathrm{Mn}_{3} \mathrm{O}_{4}, 12 \% \mathrm{LiMn}_{2} \mathrm{O}_{4}, 10 \%$ $\mathrm{LiCl}, 4 \% \mathrm{~h}-\mathrm{YMnO}_{3}$ and $2 \% \mathrm{o}-\mathrm{YMnO}_{3}$. 


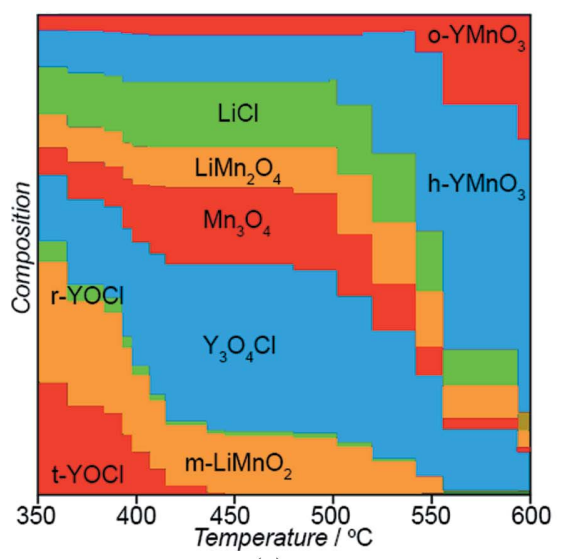

(a)

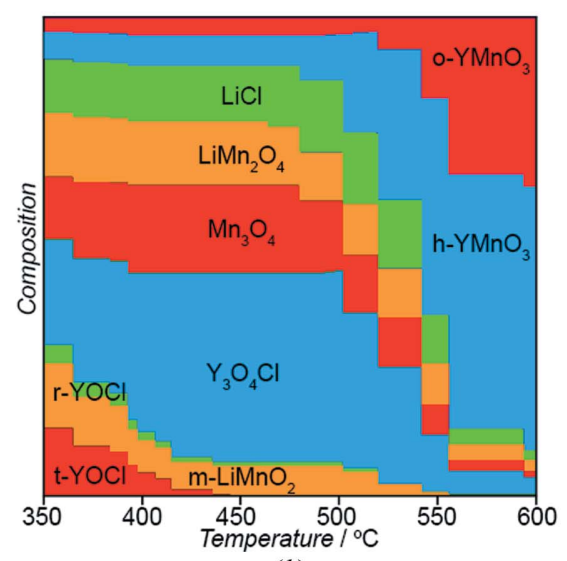

(b)

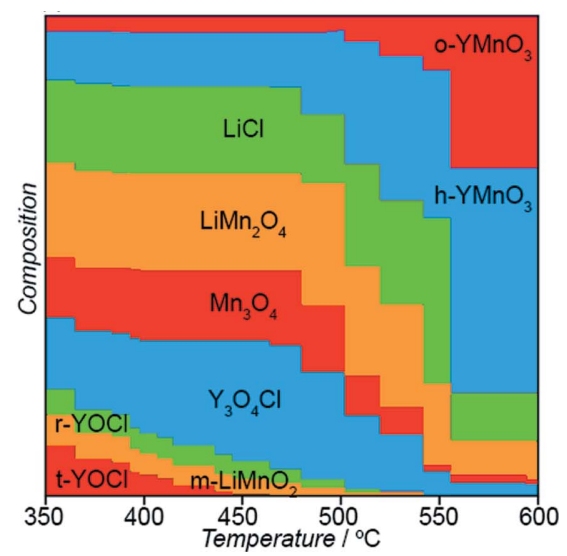

(c)

Figure 9

Phase composition identified at each position along the gradient as a function of dwell temperature, measured at three reaction times: $(a) 20$ min, $(b)$ $40 \mathrm{~min}$ and $(c)$ recovered.

observed together, that is, peaks from a given crystalline phase and other crystalline phases that form and/or disappear at the same time. It is important to note that NMF does not necessarily decompose series of data into the contributions from individual pure phases but recovers the components whose summation best describes those data. Doing so affords fewer data sets requiring analysis, thereby improving efficiency. Here, five NMF components were observed, describing nine crystallographic phases observed across the series of data.

The components from the NMF analysis include features from real crystalline phases and the evolution of the component weightings reflect the progression of intermediate reactions along the reaction pathway; Rietveld refinements were undertaken on the components in TOPAS (Fig. 8). The analysis identified two polymorphs of yttrium manganese oxide, formed at different temperatures: orthorhombic, o-YMnO $\mathrm{Yn}_{3}$ (Pnma), and hexagonal, h-YMnO $3\left(\mathrm{~Pb}_{3} \mathrm{~cm}\right)$ (Fig. 9). $\mathrm{LiMnO}_{2}(C 2 / m)$ and $\mathrm{YOCl}(P 4 / n m n)$ form intermediates r-YOCl $(R \overline{3} m)$ and the spinel $\mathrm{LiMn}_{2} \mathrm{O}_{4}$; r-YOCl then oxygenates to $\mathrm{Y}_{3} \mathrm{O}_{4} \mathrm{Cl}$. Indeed, $\mathrm{r}-\mathrm{YOCl}$ is observed in the $\mathrm{NMF}$ components despite being highly disordered (broad diffraction peaks). Lithium chloride presents an interesting, albeit not general, case for the gradient heater, as it melts at $605^{\circ} \mathrm{C}$, resulting in the absence of $\mathrm{LiCl}$ peaks in in situ data above this temperature. The spinel $\mathrm{LiMn}_{2} \mathrm{O}_{4}$ is thermodynamically favoured as $\mathrm{LiMnO}_{2}$ delithiates to form $\mathrm{LiCl}$ (Kitchaev et al., 2017). As $\mathrm{LiCl}$ reformed, we identified intermediates $\mathrm{Y}_{3} \mathrm{O}_{4} \mathrm{Cl}$ and spinel $\mathrm{Mn}_{3} \mathrm{O}_{4}$ as majority phases in the calculated components. The analysis shows the time evolution of the reaction at different temperatures, including transformation between different polymorphs; for example, an increasing proportion of o- $\mathrm{YMnO}_{3}$ relative to $\mathrm{h}-\mathrm{YMnO}_{3}$ is observed with increasing reaction time.

\section{Discussion and conclusions}

The gradient-heater tests and modelling highlight the potential to customize the temperature profile across a sample by changing the spacing of the resistive wire windings. While the present work targeted a linear monotonic gradient, alternative designs could produce a nonlinear temperature gradient or mirror the temperature gradient with the spacing of the resistive wires arranged symmetrically (this would reduce the need for spatial fiducial markers). The potential to model and rapidly prototype gradient heaters with different wire configurations facilitates such customization.

The strategies developed here to generate a controlled reproducible temperature gradient across the sample may be equally applied to deliver more uniform heating in conventional applications of the flow-cell/furnace. Specifically, 3Dprinted ceramic bars with evenly spaced grooves could facilitate uniform winding of the resistive wire which, at present, relies on the skill of the experimenter. Furthermore, the rectangular profile of the ceramic bars can both shield air currents around the sample capillary, reducing this major source of thermal fluctuations, and provide more uniform heating in the plane of the sample than cylindrical rods.

The temperature steps that can be resolved spatially across the temperature gradient are limited by the width of the X-ray beam and the slope of the temperature gradient. We note that the temperature-gradient approach can be combined with the conventional variable-temperature approach to increase the temperature resolution. By collecting a series of gradient measurements and changing the maximum temperature by less than the temperature difference between adjacent points, one can reconstruct the temperature profile with higher resolution, where different, but overlapping, data sets fill the gaps. This hybrid approach can mitigate the compromise between range and resolution for studies over wide temperature ranges.

The fidelity of the thermal-gradient approach relies on the reproducibility of the gradient and sample positioning. Large changes in the sample volume upon heating may allow the sample to move, introducing possible errors in estimated temperature. These changes will be notable during decomposition reactions, for example, where gaseous by-products are evolved. The shift of the sample position and associated errors can be minimized by ensuring that the sample powder is 
well packed and further mitigated by mixing the sample with an inert low-scattering matrix (e.g. glass wool).

Compared with conventional variable-temperature studies, where the sample can be similar in dimension to the X-ray beam, the extended region of the capillary being probed across the thermal gradient requires a larger sample volume (ca $4 \mathrm{~cm}$ cf. $4 \mathrm{~mm}$ capillary filled). However, this is still substantially less sample than required for conventional ex situ studies following treatment at different elevated temperatures.

As the entire temperature range of interest exists simultaneously, the thermal-gradient approach to variabletemperature measurements is inherently more efficient than conventional methods, eliminating the time overhead associated with changing and stabilizing the temperature. Temperature and time become independent dimensions, allowing for simultaneous in situ studies of the thermodynamic and kinetic properties of a material. By allowing multiple temperatures to be probed in parallel, the gradient heater facilitates time-dependent studies of processes (e.g. reactions, transformation, annealing) at different temperatures. Such measurements are needed to estimate kinetic parameters and to identify temperature-dependent reaction mechanisms. For example, by probing the time dependence of different reactions at several temperatures, it is possible to estimate activation energies (Zhao et al., 2011). The continuous nature of the temperature gradient means that phases present in narrow temperature windows are not overlooked as can occur for temperature measurements at a series of discrete temperature points.

The additional potential for the gradient heater to pre-treat a system at a continuum of elevated temperatures before being recovered to ambient temperature for measurements opens opportunities for new types of experiments and analysis. For example, parametric studies of systems recovered following treatment at a series of temperatures is relevant to probing annealing processes or emulating the products recovered from solid-state reactions. Undertaking such studies using a conventional approach is labour and sample intensive and, as such, they are typically limited to a handful of temperatures. Analysis of systems recovered to ambient temperature is more straightforward as it eliminates temperature dependence of the structure, for example, thermal expansion. This, in turn, may simplify the separation of phases through multivariate data analytic approaches such as principal component analysis or non-negative matrix factorization (Chapman et al., 2015; Geddes et al., 2019).

In summary, we believe the thermal-gradient approach is a new paradigm in variable-temperature studies, which is amenable to measurements of systems both at a continuum of elevated temperatures and following recovery to ambient conditions.

\section{Related literature}

The following additional literature is cited in the supporting information: Ditmars et al. (1982); Endo et al. (2003); Evans et al. (1997); Glassbrenner \& Slack (1964); Haynes (2013-2014);
Janiak (2003); Jund \& Jullien (1999); Leadbetter et al. (1969); Lee \& Seung (2001); Matsuda et al. (2004); Modest (2013); Murashov \& White (2000); Ravindra et al. (2001); Sato (1967); Shimizu et al. (2013); Wang \& Zhang (2013).

\section{Funding information}

This work was supported as part of GENESIS: A Next Generation Synthesis Center, an Energy Frontier Research Center funded by the US Department of Energy (DOE), Office of Science, Basic Energy Sciences under award No. DESC0019212. This research used beamline 11-ID-B of the Advanced Photon Source, a US DOE Office of Science User Facility operated for the DOE Office of Science by Argonne National Laboratory under contract No. DE-AC0206CH11357. This research also used beamlines 28-ID-1 and 28-ID-2 of the National Synchrotron Light Source II, a US DOE Office of Science User Facility operated for the DOE Office of Science by Brookhaven National Laboratory under contract No. DE-SC0012704.

\section{References}

Chapman, K. W., Chupas, P. J. \& Kepert, C. J. (2005). J. Am. Chem. Soc. 127, 15630-15636.

Chapman, K. W., Lapidus, S. H. \& Chupas, P. J. (2015). J. Appl. Cryst. 48, 1619-1626.

Chupas, P. J., Chapman, K. W., Kurtz, C., Hanson, J. C., Lee, P. L. \& Grey, C. P. (2008). J. Appl. Cryst. 41, 822-824.

Coelho, A. A. (2018). J. Appl. Cryst. 51, 210-218.

Ditmars, D. A., Ishihara, S., Chang, S. S., Bernstein, G. \& West, E. D. (1982). J. Res. Natl Bur. Stnd. 87, 159-163.

Drews, A. R. (2000). Adv. X-ray Anal. 44, 44-49.

Endo, R., Fujihara, Y. \& Susa, M. (2003). High Temp. High Press. 35/ 36, 505-511.

Evans, J. S. O., Mary, T. A. \& Sleight, A. W. (1997). J. Solid State Chem. 133, 580-583.

Evans, J. S. O. \& Radosavljević Evans, I. (2004). Chem. Soc. Rev. 33, 539-547.

Geddes, H. S., Blade, H., McCabe, J. F., Hughes, L. P. \& Goodwin, A. L. (2019). Chem. Commun. 55, 13346-13349.

Glassbrenner, C. J. \& Slack, G. A. (1964). Phys. Rev. 134, A1058A1069.

Griffith, K. J., Forse, A. C., Griffin, J. M. \& Grey, C. P. (2016). J. Am. Chem. Soc. 138, 8888-8899.

Haynes, W. M. (2013-2014). CRC Handbook of Chemistry and Physics, 94th ed. Boca Raton: CRC Press LLC.

Janiak, C. (2003). Dalton Trans. p. 2781.

Jiang, Z., Ramanathan, A. \& Shoemaker, D. P. (2017). J. Mater. Chem. C, 5, 5709-5717.

Jund, P. \& Jullien, R. (1999). Phys. Rev. B, 59, 13707-13711.

Kitchaev, D. A., Dacek, S. T., Sun, W. \& Ceder, G. (2017). J. Am. Chem. Soc. 139, 2672-2681.

Kusne, A. G., Keller, D., Anderson, A., Zaban, A. \& Takeuchi, I. (2015). Nanotechnology, 26, 444002.

Leadbetter, A. J., Newsham, D. M. T. \& Settatree, G. R. (1969). J. Phys. C Solid State Phys. 2, 393-403.

Lee, D. D. \& Seung, H. S. (1999). Nature, 401, 788-791.

Lee, D. D. \& Seung, H. S. (2001). Advances in Neural Information Processing Systems 13, edited by T. K. Leen, T. G. Dietterich \& V. Tresp, pp. 556-562. Cambridge: MIT Press.

Long, C. J., Bunker, D., Li, X., Karen, V. L. \& Takeuchi, I. (2009). Rev. Sci. Instrum. 80, 103902. 
Martinolich, A. J. \& Neilson, J. R. (2017). Chem. Mater. 29, 479489.

Matsuda, R., Kitaura, R., Kitagawa, S., Kubota, Y., Kobayashi, T. C., Horike, S. \& Takata, M. (2004). J. Am. Chem. Soc. 126, 1406314070.

Millange, F., Walton, R. I. \& O'Hare, D. (2000). J. Mater. Chem. 10, 1713-1720.

Modest, M. F. (2013). Radiative Heat Transfer, 3rd ed., edited by M. F. Modest, pp. 129-159. Boston: Academic Press.

Murashov, V. V. \& White, M. A. (2000). J. Mater. Sci. 35, 649-653.

Newton, M. A., Checchia, S., Knorpp, A. J., Stoian, D., van Beek, W., Emerich, H., Longo, A. \& van Bokhoven, J. A. (2019). Catal. Sci. Technol. 9, 3081-3089.

Pedregosa, F., Varoquaux, G., Gramfort, A., Michel, V., Thirion, B., Grisel, O., Blondel, M., Prettenhofer, P., Weiss, R., Dubourg, V., Vanderplas, J., Passos, A., Cournapeau, D., Brucher, M., Perrot, M. \& Duchesnay, E. (2011). J. Mach. Learn. Res. 12, 2825-2830.

Ravindra, N. M., Sopori, B., Gokce, O. H., Cheng, S. X., Shenoy, A., Jin, L., Abedrabbo, S., Chen, W. \& Zhang, Y. (2001). Int. J. Thermophys. 22, 1593-1611.
Reiss, C. A. (2019). International Tables for Crystallography, Vol. H, Powder Diffraction, 1st online ed., edited by C. J. Gilmore, J. A. Kaduk \& H. Schenk, ch. 2.6, pp. 150-155. Chester: International Union of Crystallography.

Samaragdis, P. \& Brown, J. C. (2003). IEEE Workshop on Applications of Signal Processing to Audio and Acoustics, IEEE Cat. No. 03TH8684, pp. 177-180. IEEE.

Sato, T. (1967). Jpn. J. Appl. Phys. 6, 339-347.

Shimizu, T., Matsuura, K., Furue, H. \& Matsuzak, K. (2013). J. Eur. Ceram. Soc. 33, 3429-3435.

Shoemaker, D. P., Chung, D. Y., Mitchell, J. F., Bray, T. H., Soderholm, L., Chupas, P. J. \& Kanatzidis, M. G. (2012). J. Am. Chem. Soc. 134, 9456-9463.

Stinton, G. W. \& Evans, J. S. O. (2007). J. Appl. Cryst. 40, 87-95.

Todd, P. K., Smith, A. M. M. \& Neilson, J. R. (2019). Inorg. Chem. 58 , 15166-15174.

Wang, Y. \& Zhang, Y. (2013). IEEE Trans. Knowl. Data Eng. 25, $1336-1353$

Zhao, H. Y., Nenoff, T. M., Jennings, G., Chupas, P. J. \& Chapman, K. W. (2011). J. Phys. Chem. Lett. 2, 2742-2746. 